\title{
Lessons of the month: Acute liver failure: a case close to the heart
}

\author{
Authors: Charelle Manning, ${ }^{A}$ Hameed Rafiee ${ }^{B}$ and Syed Alam ${ }^{C}$
}

\begin{abstract}
The differential diagnosis of an acute liver injury is extremely broad and can often change following initial investigations. We describe the case of a 54-year-old woman whose liver function derangement was initially attributed to alcohol excess, but in fact turned out to be cardiac in origin. We describe the underlying mechanisms and features of cardiac-related liver injury, and how the pattern of liver tests alongside appropriate imaging can help obtain the diagnosis.
\end{abstract}

KEYWORDS: acute liver failure, congestive hepatopathy, pericardial effusion, lung cancer, deranged LFTs

DOI: 10.7861/clinmed.2020-1062

\section{Case presentation}

We present a case of a 54 -year-old woman who presented with rapidly deteriorating liver function and a 4-6-week history of feeling increasingly unwell with nausea, vomiting, weight loss and progressive lethargy. In the preceding months, she also noticed a worsening cough and exertional dyspnoea. She was an ex-smoker with a 30-pack year history. She had a history of alcohol excess but had remained largely abstinent for 2 months with the exception of a 2-week relapse prior to this presentation.

On arrival she was tachycardic (heart rate of 109 beats per minute), slightly hypotensive (blood pressure of $110 / 85 \mathrm{mmHg}$ ) and tachypnoeic (respiratory rate of 18-20 breaths per minute). Pulse oximetry showed oxygen saturations of $99 \%$ on room air. She was apyrexial. Her admission bloods are shown in Table 1. Of note, she had markedly deranged liver enzymes, coagulopathy, hyponatraemia and massive hyperferritinaemia.

Electrocardiography showed a sinus tachycardia with $\mathrm{T}$ wave flattening in the inferolateral leads. Troponin was negative. Chest $X$-ray showed a left pleural effusion and suspicion of a left apical mass.

Given her history of alcohol excess and deranged liver tests, she was commenced on a detox regimen and sent to the hepatology ward. On further assessment, she appeared listless

Authors: ${ }^{\text {A }}$ senior hepatology fellow, Norfolk and Norwich University Teaching Hospital, Norwich, UK; ${ }^{\mathrm{B}}$ consultant radiologist, Norfolk and Norwich University Teaching Hospital, Norwich, UK; ' ${ }^{C}$ consultant hepatologist, Norfolk and Norwich University Teaching Hospital, Norwich, UK

\section{Table 1. Admission blood results}

\begin{tabular}{ll} 
Measurement & Value (normal range) \\
White cell count, $\times 10^{9} / \mathrm{L}$ & $12.2(4.0-10.0)$ \\
Haemoglobin, $\mathrm{g} / \mathrm{L}$ & $119(130-170)$ \\
Neutrophils, $\times 10^{9} / \mathrm{L}$ & $10.07(2.00-7.00)$ \\
Platelets, $\times 10^{9} / \mathrm{L}$ & $148(150-410)$ \\
C-reactive protein, $\mathrm{mg} / \mathrm{L}$ & $51(<10)$ \\
Sodium, $\mathrm{mmol} / \mathrm{L}$ & $125(133-146)$ \\
Potassium, mmol/L & $4.0(3.5-5.3)$ \\
Urea, $\mathrm{mmol} / \mathrm{L}$ & $10.6(1.7-7.1)$ \\
Creatinine, $\mu \mathrm{mol} / \mathrm{L}$ & $52(59-104)$ \\
Estimated glomerular filtration rate, & $>90(>60)$ \\
$\mathrm{mL} / \mathrm{min} / 1.73 \mathrm{~m}{ }^{2}$ & \\
Bilirubin, $\mu \mathrm{mol} / \mathrm{L}$ & $36(<20)$ \\
Alanine aminotransferase, $\mathrm{U} / \mathrm{L}$ & $779(<50)$ \\
Alkaline phosphatase, $\mathrm{U} / \mathrm{L}$ & $356(38-126)$ \\
Gamma glutamyl transferase, $\mathrm{U} / \mathrm{L}$ & $648(<60)$ \\
Albumin, g/L & $37(35-50)$ \\
International normalised ratio & $1.56(<1.4)$ \\
Ferritin, $\mu \mathrm{g} / \mathrm{L}$ & $11,942(23-300)$ \\
Vitamin $\mathrm{B} 12, \mathrm{ng} / \mathrm{L}$ & $>2,000(187-883)$ \\
Folate, $\mu \mathrm{g} / \mathrm{L}$ & $13.1(2.7-15.0)$ \\
\hline
\end{tabular}

and encephalopathic, with a raised jugular venous pressure and tender hepatomegaly. Further blood tests showed a worsening liver injury with an alanine aminotransferase (ALT) of 1,833 U/L, bilirubin of $52 \mu \mathrm{mol}$, INR of 2.12 and a serum ammonia of 128 $\mu \mathrm{mol} / \mathrm{L}$ confirming hepatic encephalopathy. This triad of deranged liver enzymes, coagulopathy and hepatic encephalopathy defined a clinical picture of acute liver failure (ALF). In this context, the substantially raised serum ferritin would be in keeping with a massive hepatocellular injury.

Further work-up with an urgent computed tomography (CT) of the chest, abdomen and pelvis (Fig 1) confirmed a large pericardial effusion with right ventricular collapse, inferior vena cava dilatation and bilateral pleural effusions. The liver appeared 

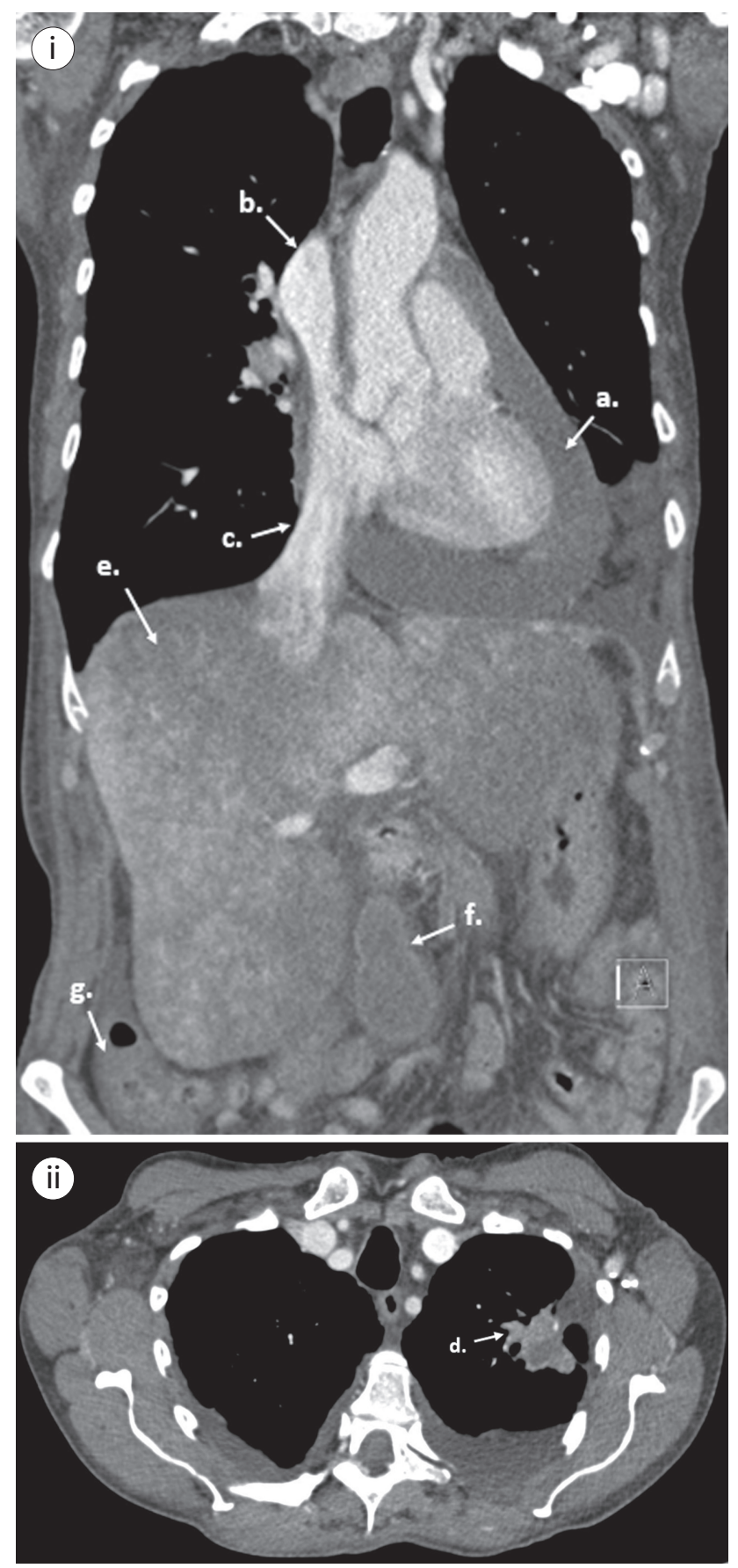

Fig 1. Post-contrast tomography of the chest, abdomen and pelvis showing a large pericardial effusion (a), superior and inferior vena cava dilatation ( $b$ and $c$ ), spiculated pulmonary mass (d), hepatic congestion (e), and oedematous gallbladder and colon ( $\mathrm{f}$ and $\mathrm{g}$ ) resulting from portal hypertension. i) Coronal plane. ii) Axial plane.

markedly oedematous, which, upon further discussion with the radiologist, was described as a classical 'nutmeg' appearance secondary to venous congestion. Unfortunately, imaging also confirmed a spiculated left upper lobe mass consistent with a primary lung cancer, a pulmonary embolism and cerebral metastases seen on $\mathrm{CT}$ of the head.
An urgent echocardiography confirmed a large $4 \mathrm{~cm}$ pericardial effusion with evidence of ventricular compromise. She underwent an emergency pericardial drain and $1.5 \mathrm{~L}$ of bloodstained fluid was removed, providing immediate relief to some of her symptoms. Subsequent fluid cytology confirmed malignant cells consistent with small cell lung cancer.

The overall clinical picture was in keeping with metastatic small cell lung cancer resulting in a haemorrhagic pericardial effusion and cardiac tamponade, leading to hepatic outflow tract obstruction which resulted in acute liver failure. Despite initial symptomatic improvement, she unfortunately succumbed to significant disease burden and died peacefully.

\section{Discussion}

This is an intriguing case of an advanced lung cancer masquerading as an acute liver failure. It is to be noted that, although she had a history of alcohol excess, the degree of transaminitis would argue against the diagnosis of alcoholic hepatitis, the most severe manifestation of alcohol-related liver disease. Such a significantly elevated ALT is rarely seen in alcoholic hepatitis and as such should prompt a search for an alternative cause. ${ }^{1}$ The differential diagnosis of an acute hepatitis with very high transaminases includes acute viral hepatitis, autoimmune hepatitis, Wilson's disease, drug-induced liver injury and hepatic ischaemia.

The current literature describes several cases of lung cancer presenting as ALF, mostly due to hepatic metastases. In our patient, although hepatic metastatic infiltration is a possibility, the clinical features as well as the classical radiological pattern would favour liver congestion as the cause of liver failure. There have been two reported cases of lung cancer causing ALF in the setting of a pericardial effusion and tamponade, both having a similar liver profile to our case, with notable improvement following pericardiocentesis. $^{2,3}$

Regarding ALF secondary to pericardial effusion of any cause, there have been a few more reported cases in the literature. ${ }^{4-7}$ The underlying mechanism of liver injury in these cases has been debated. There is evidence to suggest that low cardiac output secondary to pericardial effusion causes reduction in blood flow to the liver and subsequent hypoxic or ischaemic injury. Another consideration is that of hepatic venous congestion from right-sided heart failure. Both mechanisms may be contributary, although low cardiac output is thought to have the more dominant role. ${ }^{8}$ It is theorised that the pattern of liver function tests may be of use in differentiating the predominant aetiology, ie a hepatotoxic pattern in ischaemic injury vs a cholestatic pattern in hepatic congestion. Imaging features may help discriminate between hepatic hypoperfusion and congestive liver injury; the former typically demonstrating parenchymal hypoenhancement on contrast-enhanced imaging, whereas the classical mottled or 'nutmeg' appearance of the liver usually suggests a venous outflow obstruction. ${ }^{9,10}$ Other supportive features of congestion include dilated hepatic veins and inferior vena cava with features of right heart failure (as seen in our case). The venous congestion in the liver can also produce certain characteristic features on histology. These include dilatation of the hepatic sinusoids and subsequent perisinusoidal oedema, thrombosis and haemorrhage, with loss of hepatocytes around the central vein and eventual development of fibrosis. ${ }^{9}$ However, it is often not necessary to perform a liver biopsy in the acute setting as the clinical 
presentation and imaging findings are often diagnostic in their own right.

\section{Key points}

> Cardiopulmonary pathologies can manifest as acute liver failure and should be considered in the differential diagnosis of any patient presenting with an acute transaminitis.

> The pattern of liver function test abnormality is often the first clue as to the underlying aetiology and can help refine the differential diagnosis.

> Judicious use of imaging, particularly hepatic vasculature, can help lead to the right diagnosis.

\section{Acknowledgements}

We would like to thank the patient's family for allowing us to publish this case report.

\section{References}

1 Torruellas C, French SW, Medici V. Diagnosis of alcoholic liver disease. World J Gastroenterol 2014;20:11684-99.

2 Jamil KM, van Hagan T, Trotter J, Cheng W, Kontorinis N. Acute liver failure in a patient with lung cancer. Gut 2007:56:1190.

3 Sazgar S, Parbtani R, Thai TN, Campion JM. Cardiac tamponadeinduced severe ischemic hepatitis. American Journal of Respiratory and Critical Care Medicine 2019;199:A3536.
4 Allen C, Sanchez A, Zapatier J. Acute liver failure from cardiogenic shock without classic heart failure exam findings. American Journal of Gastroenterology 2016;111:S932.

5 Lange A, Sankey C. Individual case: Cardiac tamponade and acute liver failure. ACP Hospitalist 2019. https://acphospitalist.org/ archives/2019/01/brief-case-individual-cardiac-tamponade.htm [Accessed 07 November 2020].

6 Thaker S, Yazici C, Koppe S. Atypical presentation of cardiac tamponade as a cause of acute liver injury: case report and review of literature. Cureus 2018;10:e2779.

7 Saner $\mathrm{FH}$, Heuer M, Meyer $\mathrm{M}$ et al. When the heart kills the liver: Acute liver failure in congestive heart failure. Eur J Med Res 2009;14:541-6.

8 Nikolaou M, Mebazaa A. Cardiohepatic interactions in heart failure: clinical and therapeutic implications. Continuing Cardiology Education 2017:3:117-20.

9 Michael L, Wells ML, Fenstad ER, Poterucha JT et al. Imaging Findings of Congestive Hepatopathy. Radiographics 2016;36:1024-37.

10 Mills A, Mellnick V. Imaging features of hepatic arterial and venous flow abnormalities. Clin Liver Dis (Hoboken) 2018;11:27-32.

Address for correspondence: Dr Syed Alam, Department of Gastroenterology \& Hepatology, Norfolk and Norwich University Hospital, Colney Lane, Norwich NR4 7UY, UK. Email: syed.alam@nnuh.nhs.uk 